\title{
Phlebographic Study between Patients with MS and Control Subjects: The Ethical Profile
}

T he term and the concept of chronic cerebrospinal venous insufficiency (CCSVI) were coined by the Italian vascular surgeon Paolo Zamboni, who proposed compromised blood drainage from the central nervous system in patients with MS, caused by strictures and valves in the major veins of the neck.

Very soon after the description in 2009 of CCSVI as the major, if not unique, causal factor of MS, ${ }^{1}$ uncontrolled angioplastic or stent placement procedures in the jugular veins, initially termed "liberation therapies," were increasingly offered to patients with the disease. The "CCSVI community" grew rapidly on the Web and gained the attention of the media and of the general public. As a result of misunderstanding and oversimplification, not only patients with MS but also patients with other neurodegenerative disorders such as amyotrophic lateral sclerosis decided to undergo the angioplastic procedure, even if discouraged by their doctors.

In their first publication, Zamboni et $\mathrm{al}^{1}$ described venous abnormalities, identified through combined transcranial and extracranial color Doppler high-resolution examination (TCCS-ECD) and confirmed by selective venography of the azygous and jugular venous system, to be dramatically associated with MS only because they were never detectable in healthy individuals or in subjects with other neurologic diseases. In response to criticisms coming from other research groups who failed to replicate the TCCS-ECD findings of Zamboni et al (including the authors of the study published in this issue of the American Journal of Neuroradiology and Centonze et $\mathrm{al}^{2}$ ), the CCSVI community proposed the argument that CCSVI detection through TCCS-ECD is a very tough task, requiring appropriate training and specific equipment. In the absence of specific training, operator abilities, and the proper TCCS-ECD machine, only objective venographic evaluations were claimed by Zamboni et $\mathrm{al}^{1}$ to be suitable to detect CCSVI.

In this atmosphere, the proposal coming from the authors of this study to perform selective venography in patients with MS appeared to me and to the other members of the ethical committee of my institution crucial to provide a final answer to the CCSVI scientific debate, but only if combined with blind venographic studies in the appropriate control subjects. Proper controls were not investigated by Zamboni et al or by others, and in fact the 2009 study of Zamboni et al ${ }^{1}$ only reported unblinded results in control subjects who underwent the phlebographic procedure but were not matched with the patients with MS.

In general terms, experimental science cannot do without experimentation due to intrinsic epistemologic requirements. In particular, experimentation is essential for the progress of medical science. As stated in Human Rights and Biomedicine ${ }^{3}$, medical progress is based on research, an essential part of which is the realization of studies that include human subjects. In these studies, however, the interests and welfare of the human being will prevail over the sole interest of society or science.

The ethical values regarding experimentation on human beings are, in hierarchical order: the defense of life and of the person, which is the key point of ethics; the validity of the therapeutic principle; and the social theme connected to scientific progress. In accordance with the principle of social solidarity, it is legitimate to ask an individual to share in the risk for the good of the entire society of which he or she is a member, keeping in mind, however, that there is, in the individual, the global value and fundamental reason of existence of society itself.

In experimentation on healthy volunteers, such a principle is applied that legitimizes experimentation on the basis of the following conditions:

1) The first condition is that the consensus must be free, explicitly informed, and personal and regard a healthy (not presumed to be) individual who is not connected by work or position to the person conducting the experiment;

2) The risk that the volunteer is subjected to must not exceed the limit of the defense of the life and integrity of that person. Within these limits, the principle of proportionality between risks and benefits is always in force: clinical studies of insufficient or doubtful scientific validity cannot be ethically accepted. The experimenter must always be ready to interrupt the experiment in the event that the informed consensus is withdrawn or unforeseen risks arise.

The invasiveness of the phlebographic procedure for patients and healthy individuals was well taken into account when the 
clinical research plan was approved by our institutional ethical committee. Nonetheless, the advancements in knowledge stemming from well-designed and conducted research on CCSVI were finally judged to beneficially affect the lives of thousands of patients with MS and of their doctors, who rely on scientific evidence to make their decisions.

\section{REFERENCES}

1. Zamboni P, Galeotti R, Menegatti E, et al. Chronic cerebrospinal venous insufficiency in patients with multiple sclerosis. J Neurol Neurosurg Psychiatry 2009;80:392-99
2. Centonze D, Floris R, Stefanini M, et al. Proposed chronic cerebrospinal venous insufficiency criteria do not predict multiple sclerosis risk or severity. Ann Neurol 2011;70:51-58

3. Convention on Human Rights and Biomedicine. Oviedo, Spain, April 4, 1997. http://conventions.coe.int/Treaty/en/Treaties/ Html/164.htm

M. G. Marcian

Professor of Neurology and President of Ethical Committee of the Fondazione Policlinico Tor Vergata, Rome, Italy

http://dx.doi.org/10.3174/ajnr.A3805 